\title{
Prognostic Value of Serum Neutrophil Gelatinase-Associated Lipocalin in Metastatic and Non-metastatic Colorectal Cancer: Reply
}

\author{
Josep Marti $\cdot$ Josep Fuster
}

Published online: 26 July 2013

(C) Société Internationale de Chirurgie 2013

We read with pleasant interest the letter from Dr. Kapoor commenting on articles related to our recently published article in the World Journal of Surgery about the prognostic value of serum neutrophil gelatinase-associated lipocalin (NGAL) levels in regard to metastatic and nonmetastatic colorectal cancers [1].

As we mentioned in our article, several studies have been designed to ascertain the exact role of NGAL in neoplastic disease, but this role remains unclear. Nevertheless, given the increasing number of studies that point to NGAL as a key element in the progression of various cancers, we agree with Dr. Kapoor that a rising research interest about the role of NGAL on tumor progression can be expected during the next few years.
We thank Dr. Kapoor for his kind and encouraging comments about our article's topic. We hope that our research team can offer further interesting results regarding the role of NGAL in tumor progression in the near future.

\section{Reference}

1. Martí J, Fuster J, Solà AM et al (2013) Prognostic value of serum neutrophil gelatinase-associated lipocalin in metastatic and nonmetastatic colorectal cancer. World J Surg 37:1103-1109. doi:10. 1007/s00268-013-1930-z

J. Marti · J. Fuster $(\bowtie)$

Liver and Surgery and Transplantation Unit,

Hospital Clinic i Provincial, Barcelona, Spain

e-mail: jfuster@clinic.ub.es

J. Marti

e-mail: josepmartis@yahoo.es 